\title{
Aurora kinase inhibitors: Potential molecular-targeted drugs for gynecologic malignant tumors (Review)
}

\author{
KIYOKO UMENE, KOUJI BANNO, IORI KISU, MEGUMI YANOKURA, YUYA NOGAMI, KOSUKE TSUJI, \\ KENTA MASUDA, ARISA UEKI, YUSUKE KOBAYASHI, WATARU YAMAGAMI, HIROYUKI NOMURA, \\ EIICHIRO TOMINAGA, NOBUYUKI SUSUMU and DAISUKE AOKI
}

Department of Obstetrics and Gynecology, School of Medicine, Keio University, Tokyo 160-8582, Japan

Received January 21, 2013; Accepted March 12, 2013

DOI: $10.3892 / b r .2013 .91$

\begin{abstract}
Chemotherapy and surgery are important treatment strategies for gynecologic malignant tumors such as ovarian, cervical and endometrial cancer. However, many anticancer drugs currently available are cytotoxic and cause strong adverse reactions in patients. Aurora kinases have attracted increasing attention in recent years as serine/threonine kinases with various roles in cell division, including chromosomal agglutination and segregation, functions of centromeres, centrosomal maturation, spindle formation and cytokinesis. Aurora kinases are overexpressed in a number of cancers and recent studies have shown that they are involved in oncogenesis and cause an aberrant increase in centrosome number, emergence of polykaryocytes and failure of cancer inhibition mechanisms. Thus, drugs that inhibit Aurora kinases are likely to exert anticancer effects in various fields, including the gynecologic field. Aurora kinase inhibitors exert antitumor effects in monotherapy and synergistic effects in combination therapy with taxane-based anticancer agents for gynecologic tumors and are likely to increase the efficacy of existing anticancer drugs. Current Aurora kinase inhibitors include ZM447439, Hesperadin, VX-680/MK-0457, AT9283 and Barasertib, and clinical trials are ongoing to verify the effects of these inhibitors.
\end{abstract}

\section{Contents}

1. Introduction

2. What are Aurora kinases?

3. Clinical applications of Aurora kinase inhibitors

4. Gynecologic applications of Aurora kinase inhibitors

5. Conclusion

Correspondence to: Dr Kouji Banno, Department of Obstetrics and Gynecology, School of Medicine, Keio University, Shinanomachi 35, Shinjuku-ku, Tokyo 160-8582, Japan

E-mail: kbanno@z7.keio.jp

Key words: Aurora kinase A, Aurora kinase inhibitor, gynecologic cancer, hesperadin, VX-680, ZM447439

\section{Introduction}

Conventional anticancer agents reduce the size of tumors by damaging cells. However, the targeted cells are not specific, leading to damage to normal cells and adverse reactions. Therefore, cytotoxic anticancer drugs are administered at a maximum tolerated dose calculated based on the anticancer effect and adverse effects. By contrast, molecular-targeted anticancer agents are developed based on the analysis of differences between cancer and normal cells at the whole genome or molecular level as drugs that inhibit specific molecules and which cause cancer proliferation or metastasis. Thus, these agents have more specific effects on cancer cells and a much lower level of adverse reactions than conventional anticancer drugs, permitting administration at the minimum effective dose, rather than the maximum-tolerated dose. This is a major advantage of molecular-targeted anticancer agents. However, conventional anticancer drugs currently play a key role in treatments such as neoadjuvant and adjuvant chemotherapy for gynecologic malignant tumors including ovarian, cervical and endometrial cancer. These approaches use monotherapy or multidrug combination therapy, mainly with platinum-containing agents or taxanes. However, many patients suffer from associated adverse reactions, which has led to a search for more effective drug combinations. Investigations of the antitumor effects of molecular-targeted drugs for gynecologic tumors are also underway, including for everolimus, an inhibitor of mammalian target of rapamycin and bevacizumab, a vascular endothelial growth factor inhibitor.

\section{What are Aurora kinases?}

Correct replication and distribution to daughter cells are essential for genetic information to be correctly inherited by the daughter cells. Normal cell division requires regulation by a plurality of protein kinases that each act with temporal and spatial correctness. These mitotic kinases include checkpoint-associated kinases such as Cyclin B-CDK1, as well as NimA, Polo, Aurora and WARTS kinases. The transition from $\mathrm{G} 2$ to $\mathrm{M}$ phase is the major control point in cell division and loss of function of these kinases causes chromosomal instability due to failed division and subsequent oncogenesis (1-4). 
Table I. Properties of Aurora kinases.

\begin{tabular}{lcll}
\hline Kinases & Chromosomal location & Expression in cancer & \\
\hline Aurora A & $20 \mathrm{q} 13.2$ & Amplification & Function/role \\
& & Overexpression & $\begin{array}{l}\text { Targeting to microtubules } \\
\text { Suppression of transformation potential } \\
\text { Inhibition of kinase activity } \\
\text { Positive and negative regulation of kinase activity } \\
\text { Control of microtubule dynamics at the spindle poles }\end{array}$ \\
& & Overexpression & Targeting to centromere/midzone \\
& & & $\begin{array}{l}\text { Elevation of kinase activity } \\
\text { Chromosome segregation? } \\
\text { Aurora B }\end{array}$ \\
& & & Cytokinesis? \\
& & & Inhibition of kinase activity \\
Aurora C & & Unclear
\end{tabular}

Aurora kinase genes were first isolated in 1995 from a Drosophila mutant that showed abnormal spindle formation in $\mathrm{M}$ phase. Aurora kinases are serine/threonine kinases associated with the regulation of cell division in the G2-M phases, and are particularly associated with chromosomal agglutination and segregation, functions of centromeres, centrosomal maturation, spindle formation and cytokinesis. The human Aurora kinase family comprises the members Aurora kinase A (AURKA), B (AURKB) and C (AURKC) (Table I). All three members have a common $\mathrm{N}$-terminal domain of various lengths, a kinase domain and a short C-terminal domain. However, they have a different location and timing of action and are considered to contribute independently to the progression of $\mathrm{M}$ phase. AURKA contains a catalytic kinase domain, an activating T-loop, a destruction box (D-box) and a D-box-activating domain (DAD). The D-box and DAD regions are degraded at the last stage of mitosis. AURKB and AURKC do not contain a DAD and the D-box is not degraded (Fig. 1) (1).

AURKA accumulates at centrosomes during the late G2 phase and partially moves into the nucleus before the cell enters $\mathrm{M}$ phase, and before accumulating in the nucleus during $M$ phase. Subsequent to the breakdown of the nuclear envelope, AURKA accumulates at spindle poles and on spindles due to segregation of the centrosomes replicated during $\mathrm{S}$ phase. Targeting protein for Xenopus kinesin-like protein 2 (TPX2) is an important activator of AURKA. AURKA activated by TPX2 is introduced into microtubules and is associated with centrosomal functions such as maturation and the formation of microtubules through replication, formation and stabilization of spindles in the metaphase. In late $\mathrm{M}$ phase, the amount of AURKA is downregulated by ubiquitin-mediated degradation and AURKA moves to the central spindle present between two nuclei after segregation and disappears (1-4).

Inhibition of the expression or activity of AURKA causes delayed entry into $M$ phase and failed centrosomal segregation, resulting in the spindle not being formed. By contrast, the overexpression of AURKA causes increased centrosomal numbers in the cell or formation of aneuploid cells and poses a major risk of carcinogenesis. The gene encoding AURKA is localized to chromosome $20 \mathrm{q} 13$, which is amplified in malignant tumors such as breast and ovarian cancer and is considered to be an oncogene (5). The spindle formation checkpoint, which inhibits entry into the anaphase until the chromosomes are arranged in the equatorial plane, is also disrupted by the overexpression of AURKA. Bub1-3 and Mad1-3 play an important role at this checkpoint by managing the number of microtubules extending from the two poles at the centrosomes and attaching to daughter centromeres and pulling equally on both sides, as well as by stopping cell division when an abnormal event occurs. If activity of Bub1-3 or Mad1-3 is inhibited, cell division passes the checkpoint and enters anaphase. In cells overexpressing AURKA and with the expression of a spindle formation checkpoint protein, Mad2, Anand et al (6) reported the improper entry into anaphase as well as the progression of cell division, with the emergence of aneuploid cells. Overexpression of AURKA also causes downregulation of p53 through its kinase activity. Thus, tumor cells avoid the induction of p53-dependent apoptosis and extinction of aneuploid cells through the $\mathrm{Rb} / \mathrm{p} 16$ cellular aging pathway (7). If DNA damage or aberrant cell division occurs, p53 interacts with a transcriptional coactivator, the heterogeneous nuclear ribonucleoprotein K (hnRNPK) and induces transcription of the p53 gene. AURKA deactivates hnRNPK by phosphorylation of Ser379 in hnRNPK and indirectly inhibits p53 activity (8).

AURKB emerges in the nucleus in the early $\mathrm{S}$ phase; localizes in centromeres with three proteins, inner centromere protein, survivin and borealin, in metaphase; moves to the center of the spindle in anaphase; and regulates cytokinesis in the median zone domain of a cell undergoing mitosis. Proteins that are transported from the chromosome to the center of the spindle are referred to as chromosomal passenger proteins and have key roles in centromeres. These proteins include deletion of improper attachments that interrupt even tension on spindle fibers on both sides and induction of correct bipolar attachment and arranging of chromosomes in the equatorial plane. In cases in which microtubules are unevenly attached to a centromere, the spindle formation checkpoint is activated and transition to anaphase is inhibited. The mechanism by which AURKB enhances bipolar attachment is unclear. AURKB has 
Table II. Properties of Aurora kinase inhibitors.

\begin{tabular}{|c|c|c|c|c|}
\hline Drug & Chemical class & Profile & Other targets & Toxicity \\
\hline ZM447439 & Quinazoline derivative & $\mathrm{B}>\mathrm{A}$ & MEK, SRC, LCK & \\
\hline Hesperadin & Indolinone & $\mathrm{B}>\mathrm{A}$ & Not reported & \\
\hline VX-680/MK-0457 & 4,6-diaminopyrimidine & $\mathrm{A}=\mathrm{B}=\mathrm{C}$ & FLT3 & $\begin{array}{l}\text { Grade 4: Neutropenia } \\
\text { Grade 3: Herpes Zoster infection, } \\
\text { Others: Vomiting, diarrhea, fatigue }\end{array}$ \\
\hline AT9283 & Pyrazole derivative & Unknown & $\begin{array}{c}\mathrm{JAK} 2,3, \mathrm{BCR}-\mathrm{ABL} \\
\mathrm{V}-\mathrm{ABL}\end{array}$ & $\begin{array}{l}\text { QT prolongation (trial halted), } \\
\text { Grade3: Febrile neutropenia }\end{array}$ \\
\hline AZD1152 & Quinazoline derivative & $\mathrm{B} \gg>>A, C$ & Not reported & $\begin{array}{l}\text { Grade3/4: Neutropenia, } \\
\text { Grade1/2: Diarrhea, vomiting, } \\
\text { nausea, lost appetite, hypertension }\end{array}$ \\
\hline
\end{tabular}

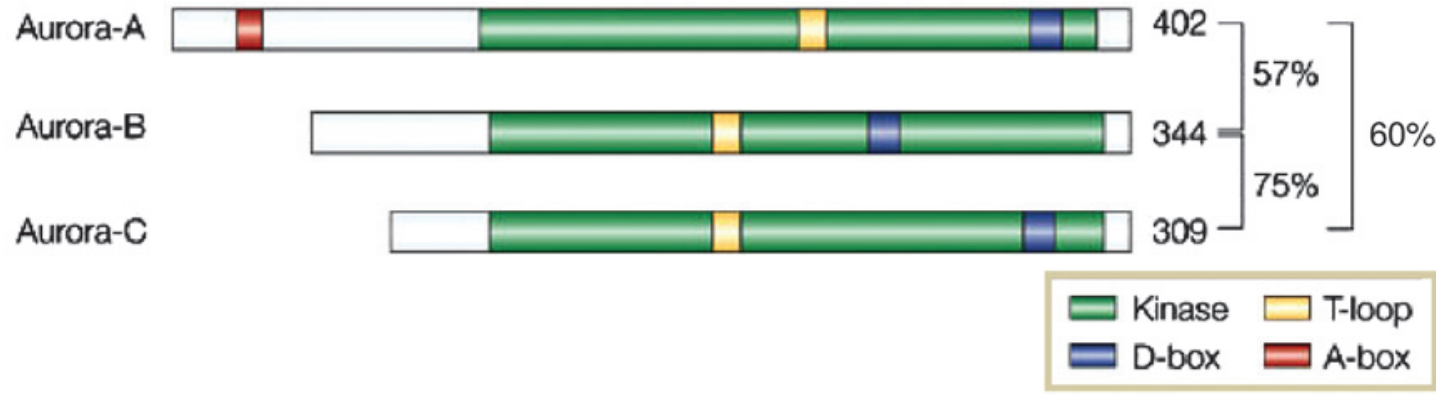

Figure 1. Human Aurora kinase family. The numbers on the right indicate sizes in amino acids and percentage sequence identities.

also been associated with chromosomal stabilization, such as chromosomal agglutination (1-3,9).

The AURKC gene is located on human chromosome 19q13.43. Recombination and deficiency are often noted in this domain in various cancer types. Overexpression of AURKC is present in many types of human cancer cells and mRNA and protein of AURKC are also highly expressed in normal tissues, particularly in the testis. AURKC-knockout mice are sterile due to abnormalities in the shape and functions of sperm and AURKC is thought to have specific actions in the formation of sperm, beyond those of AURKA and AURKB. However, compared with the other two Aurora kinases, little is known about the association of AURKC with carcinogenesis and only a few AURKC inhibitors have been developed (10).

\section{Clinical applications of Aurora kinase inhibitors}

Overexpression of Aurora kinases has been identified in many human cancer-derived cultured cell lines and cancer tissues. The amplification and overexpression of AURKA occurs in breast, bladder, colon, ovarian, pancreatic and stomach cancers. The overexpression of AURKB has been found in colon cancer, while AURKC expression occurs in various cancer-derived cell lines, including those from colon cancer, as well as in testis in normal tissues. These results suggest that the overexpression of Aurora kinases is strongly associated with tumorigenesis. For this reason, inhibitors of Aurora kinases (Table II) are being developed as potential anticancer drugs and many of these agents are in phase I and II clinical trials. These drugs only affect cells undergoing mitosis. However, many are AURKB inhibitors that may interrupt chromosome stability by AURKB and induce DNA damage, suggesting that examination of safety is important for these agents.

ZM447439 was reported as the first Aurora kinase inhibitor in 2003 and is currently in phase I clinical trials. ZM447439 inhibits the phosphorylation of histone H3 (Ser $10,28)$ by Aurora kinases. Li et al (11) showed a concentration- and time-dependent increase in the apoptotic effect of ZM447439 in a human colon cancer-derived cell line, HCT-116. ZM447439 targets the ATP-binding site common to the Aurora kinase family and theoretically should inhibit all Aurora kinases. In vitro, ZM447439 inhibits AURKA and AURKB, however, in vivo results show the predominant inhibition of AURKB, thus ZM447439 is now considered to be an AURKB inhibitor. ZM447439 causes aberrant cell division by inhibiting AURKB, inducing a p53-dependent apoptotic pathway and apoptosis of mitochondria by caspase 3 in the presence of Bak and Bax. Hesperadin is similar to ZM447439 in that it is a strong inhibitor of ATP binding to AURKB, as well as an inhibitor of $\sim 25$ other kinases. The reason for many ATP-binding site inhibitors exerting stronger effects on AURKB remains unclear (12).

VX-680 is an ATP-binding site inhibitor reported by Harrington et al (13). VX-680 is an inhibitor of AURKA, AURKB and AURKC, but exerts the strongest inhibi- 
tion on AURKB. VX-680 has shown potential as a new molecular-targeted drug for BCR/ABL-positive chronic myelogenous leukemia (CML) harboring a T315I mutation resistant to the BCR/ABL inhibitor, imatinib (13-16). However, phase II trials of VX-680 for CML harboring a T315I mutation and for Philadelphia chromosome-positive acute lymphatic leukemia (Ph+ALL) and a phase I trial for progressive leukemia, were discontinued in 2007 due to prolongation of the QT interval on electrocardiograms. Subsequently, VX-680 was modified to yield MK-0457, for which a phase I trial has been completed (17). MK-0457 also binds to the ATP-binding site and is metabolized by hepatic CYP3A4, CYP2C8 and flavin monooxygenase. In 2011, Traynor et al (18) performed a phase I trial of MK-0457 in solid cancers and demonstrated that MK-0457 is a strong inhibitor of AURKB and also interacts with the Flt-3 and Abl kinases. MK-0457 produced an outcome of stable disease in approximately half of the patients, indicating the efficacy of this agent. However, grade 4 neutropenia, grade 3 herpes infection and adverse reactions including nausea, vomiting, diarrhea and fatigue occurred during the administration period. PHA-739358 (danusertib) is also a pan-Aurora kinase inhibitor, i.e., an inhibitor of AURKA, AURKB and AURKC, similar to VX-680 and MK-0457, as well as an inhibitor of ABL and Ret tyrosine kinases. PHA-739358 has shown efficacy in clinical trials in progressive solid cancers and BCR/ABL-positive leukemia (CML, Ph+ALL) (19).

AT9283 is a synthetic heterocyclic molecule that inhibits cancer cell proliferation in solid tumors and leukemia. AT9283 inhibits AURKA and AURKB and also targets serine/threonine kinases such as JAK2, JAK3 and mutant V-abl. In 2012, Arkenau et al (20) performed a phase I study of AT9283 in patients with progressive solid cancer, in which Grade 3 pyrogenic neutropenia occurred, but major toxicities of reversible, dose-dependent bone-marrow suppression, gastrointestinal disorder, fatigue and dehairing were tolerable.

AZD1152 (barasertib) is a pro-drug that is converted to the therapeutically active hydroxyquinazoline pyrazole anilide of barasertib (barasertib-hQPA) in the cytoplasm. Barasertib-hQPA inhibits both AURKA and AURKB, although the dominant effect exerted is on AURKB. Inhibition of AURKB leads to aberrant cell division and generation of atypical cells with DNA tetraploidy or larger, which then undergo apoptosis due to checkpoint regulation. In 2012, Schwartz et al (21) reported a phase I trial of barasertib in patients with malignant solid cancer, in which Grade 3 or higher pyrogenic or apyrogenic neutropenia and mild or moderate hematoxicity or gastrointestinal toxicity emerged, but all were tolerable. In a mouse model of hepatoma, Aihara et al (22) reported that AZD1152 significantly decreased the number of tumor cells and suppressed tumor proliferation.

MLN8054 and MLN8237 (alisertib) were developed as selective AURKA inhibitors. In a phase I clinical trial of MLN8054 in progressive solid cancer, somnolence was the dose-limiting toxicity (23). This may be because the drug has a similar structure to that of benzodiazepine. Clinical trials of MLN8237 in progressive solid cancer, acute myelogenous leukemia and hematopoietic organ tumors such as non-Hodgkin's lymphoma are ongoing and a phase III trial in refractory peripheral T-cell lymphoma has recently been initiated. MLN8237 also increases cisplatin-induced apoptosis in esophageal adenocarcinoma cells (24) and enhances radiosensitivity in teratoid tumor cells (25).

\section{Gynecologic applications of Aurora kinase inhibitors}

Aurora kinases are expressed in various gynecologic malignant tumors. Li et al (26) and Kurai et al (27) examined correlations among the expression levels of AURKA and AURKB and prognosis in normal endometrium, benign tumors of the corpus uteri and endometrial cancer. The results showed significantly increased AURKA and AURKB levels in endometrial cancer compared with those in normal endometrium during the proliferation phase. AURKB was especially highly expressed in poorly differentiated endometrial cancer and AURKA and AURKB levels were correlated with worsened prognosis. Moreno-Bueno et al (28) found a high AURKA expression in type 2 non-endometrioid cancer among endometrial cancers and suggested that spindle formation checkpoint disorder in mitosis is probably involved in onset of this type of cancer. Yang et al (29) found that AURKA may be an adverse prognostic factor in ovarian endometrioid cancer, in addition to BRCA2.

Chemotherapy with taxanes or platinum-containing anticancer drugs and radiotherapy currently play key roles in the treatment of gynecologic malignant tumors. Combination of these regimens with Aurora kinase inhibitors is likely to produce synergistic effects that may increase cellular sensitivity to anticancer drugs and radiation and suppress the number of Aurora kinase inhibitor-resistant cells. For example, taxanes, which exert an anticancer effect by inhibition of depolymerization of microtubules, have increased effects on cancer cells when given in combination with Aurora kinase inhibitors. Microtubules consist of 10,000-100,000 tubulin subunits. In cells during the interphase, hundreds of long microtubules are present in the cytoplasm and connect different areas in the cell. In cell skeletons including microtubules, the subunits repeatedly associate and dissociate. Taxanes such as paclitaxel bind to microtubules and inhibit the dissociation of tubulin subunits. Inhibition of microtubule depolymerization by paclitaxel in tumor cells prevents the reconstruction of microtubules and formation of the spindle, generating aberrant cell division. The spindle formation checkpoint recognizes this abnormality and triggers apoptosis of the tumor cell, causing the tumor to decrease in size (1-4). Overexpression of Aurora kinases induces dysfunction of checkpoints in cell division and permits the cell to enter anaphase in an improper state. Thus, in the presence of overexpressed Aurora kinases, taxane-based anticancer agents are not able to induce apoptosis of aberrant cells, resulting in reduced sensitivity (6). Conversely, drugs that inhibit Aurora kinases may suppress resistance to apoptosis induced by taxanes and enhance antitumor action.

Since microtubule inhibitors such as taxanes may also affect the microtubule network of normal nerve cells and cause peripheral neuropathy, administration at a low dose is required to suppress adverse reactions. Thus, inhibition of the activity of AURKA may increase the efficacy of taxanes to the extent that the minimum effective dose can be reduced and adverse reactions decreased. Lin et al (30) examined the ovarian cancer growth-inhibitory effect of MK-0457, which inhibits AURKA, AURKB and AURKC, in mouse models. 
The results showed that MK-0457 administered alone or in combination with docetaxel significantly suppressed tumor growth by induction of apoptosis, compared with controls and that this effect was much greater for the combination regimen. In their study, Beussel et al (31) demonstrated that the response of the International Federation of Gynecology and Obstetrics surgical stage III serous ovarian cancer to first-line chemotherapy using taxanes following optimal debulking surgery could be predicted based on the expression level of AURKB.

The combination of platinum-containing anticancer agents with Aurora kinase inhibitors is also likely to increase anticancer effects. Cisplatin binds to the N7 position of guanine and adenine bases in DNA and forms crosslinks in the DNA with loss of two chlorine atoms. Thus, replication of DNA is suppressed and cell division is inhibited, providing an antitumor effect. As described above, aberrant cell division caused by Aurora kinase inhibitors leads to p53 activation and triggering of cell apoptosis. However, p53 is inhibited in a number of gynecologic tumors, including the viral protein E6 in cases with human papillomavirus (HPV) infection. However, in the cervical cancer cell line SiHa, Aurora kinase inhibitors have been shown to block the expression of HPV 16E6 and BCL-2, suppressing p53 and increasing the expression of p53, thereby increasing sensitivity to cisplatin. Therefore, enhanced effects and reduced adverse reactions of platinum-containing anticancer agents used for gynecologic tumors employ combination regimens with Aurora kinase inhibitors (32).

In cultured ovarian cancer cells, inhibition of the Aurora kinase may increase sensitivity to anticancer drugs via $\mathrm{NF}-\kappa \mathrm{B}$. In human lung cancer cells and the human ovarian cancer cell line SKOV3, which express $\mathrm{NF}-\kappa \mathrm{B}$ at high levels and are resistant to cytotoxic anticancer agents such as adriamycin and etoposide, Sun et al (33) showed that Aurora kinase inhibitors caused the downregulation of $N F-\kappa B$ and an increased anticancer effect. Aurora kinases inactivate $\mathrm{I} \kappa \mathrm{B} \alpha$ by phosphorylation, which in turn activates NF- $\kappa \mathrm{B}$ and thus Aurora kinase inhibitors suppress the inactivation of $\mathrm{I} \kappa \mathrm{B}$ and increase the drug sensitivity of cancer cells. This mechanism was verified in vitro (33) and suggests that Aurora kinase inhibitors improve chemotherapy regimens by overcoming a potential drug resistance mechanism of cancer cells.

Combination of Aurora kinase inhibitors with radiotherapy may also enhance treatment. For example, Tao et al (34) found that administration of an AURKB inhibitor, AZD1152, prior to irradiation increased the radiosensitivity of cells in which the activity of p53 was reduced by an inhibitor (pifithrin- $\alpha$ ) and of p53(-/-) mice, compared with radiation alone.

\section{Conclusion}

At present, a number of new Aurora kinase inhibitors are being developed to target malignant tumors. However, proper utilization of these inhibitors requires determination of the expression levels of Aurora kinase in each type of malignant tumor. In addition, since cancer cells often acquire resistance to low-molecular-weight agents, as seen in the emergence of imatinib-resistant CML, it is important to examine mechanisms of drug resistance to Aurora kinase inhibitors and to develop methods to overcome this resistance. Emerging shortand long-term toxicities also require evaluation as Aurora kinases play important roles in normal cells. In particular, since Aurora kinase inhibitors target the cell division system, study of the risk of secondary carcinogenesis is imperative prior to the long-term use of these inhibitors. Development of numerous Aurora kinase inhibitors is likely to increase the number of selectable drugs during treatment and contribute to the growth of tailor-made chemotherapy in which drugs are selected based on the individual patient and characteristics of the cancer during progression. Thus, the introduction of new molecular-targeted agents is likely to diversify the treatment options for intractable gynecologic cancers.

\section{Acknowledgements}

The authors gratefully acknowledge grant support from the Japan Society for the Promotion of Science (JSPS) through a Grant-in-Aid for Scientific Research (KAKENHI), a Grant-in-Aid for Scientific Research (B) (22390313), a Grant-in-Aid for Scientific Research (C) (22591866) and a Grant-in-Aid for Young Scientists (B) (24791718); the Ichiro Kanehara Foundation; Kobayashi Foundation for Cancer Research; Keio University Grant-in-Aid for Encouragement of Young Medical Scientists and the Keio University Medical Science Fund through a Research Grant for Life Sciences and Medicine.

\section{References}

1. Katayama H, Brinkly WR and Sen S: The Aurora kinases: role in cell transformation and tumorigenesis. Cancer Metastasis Rev 22: 451-464, 2003.

2. Agnese V, Bazan V, Fiorentino FP, et al: The role of Aurora-A inhibitors in cancer therapy. Ann Oncol 6: vi47-vi52, 2007.

3. Carmena $M$ and Earnshaw WC: The cellular geography of aurora kinases. Nat Rev Mol Cell Biol 4: 842-854, 2003.

4. Glover DM, Leibowitz MH, McLean DA and Parry H: Mutations in aurora prevent centrosome separation leading to the formation of monopolar spindles: Cell 81: 95-105, 1995.

5. Chung CM, Man C, Jin Y, et al: Amplification and overexpression of aurora kinase A (AURKA) in immortalized human ovarian epithelial (HOSE) cells. Mol Carcinog 43: 165-174, 2005.

6. Anand S, Penrhyn-Lowe S and Venkitaraman AR: AURORA-A amplification overrides the mitotic spindle assembly checkpoint, inducing resistance to Taxol. Cancer cell 3: 51-62, 2003.

7. Zhang D, Shimizu T, Araki N, et al: Aurora A overexpression induces cellular senescence in mammary gland hyperplastic tumors developed in p53-deficient mice. Oncogene 27: 4305-4314, 2008.

8. Hsueh KW, Fu SL, Huang CY and Lin CH: Aurora-A phosphorylates hnRNPK and disrupts its interaction with p53. FEBS Lett 585: 2671-2675, 2011.

9. Santaguida S, Vernieri C, Villa F, Ciliberto A and Musacchio A: Evidence that Aurora B is implicated in spindle checkpoint signalling independently of error correction. EMBO J 30: 1508-1519, 2011.

10. Kimmins S, Crosio C, Kotaja N, et al: Differential functions of the Aurora B and Aurora $\mathrm{C}$ kinases in mammalian spermatogenesis. Mol Endocrinol 21: 726-739, 2007.

11. Li M, Jung A, Ganswindt U, Marini P, et al: Aurora kinase inhibitor ZM447439 induces apoptosis via mitochondrial pathways. Biochem Pharmacol 79: 122-129, 2010.

12. Hauf S, Cole RW,LaTerra S, et al: The small molecule Hesperadin reveals a role for Aurora B in correcting kinetochore-microtubule attachment and in maintaining the spindle assembly checkpoint. J Cell Biol 161: 281-294, 2003.

13. Harrington EA, Bebbington D, Moore J, et al: VX-680, a potent and selective small-molecule inhibitor of the Aurora kinases, suppresses tumor growth in vivo. Nat Med 10: 262-267, 2004.

14. Gora-Tybor J and Robak T: Targeted drugs in chronic myeloid leukemia. Current Med Chem 15: 3036-3051, 2008. 
15. Young MA, Shah NP, Chao LH, et al: Structure of the kinase domain of an imatinib-resistant Abl mutant in complex with the Aurora kinase inhibitor VX-680. Cancer Res 66: 1007-1014, 2006.

16. Mancini M, Aluigi M, Leo E, et al: Histone H3 covalent modifications driving response of BCR-ABL1 ${ }^{+}$cells sensitive and resistant to imatinib to Aurora kinase inhibitor MK-0457. Br J Haematol 156: 265-268, 2012.

17. Tyler RK, Shpiro N, Marquez R and Eyers PA: VX-680 inhibits Aurora A and Aurora B kinase activity in human cells. Cell Cycle 6: 2846-2854, 2007.

18. Traynor AM, Hewitt M, Liu G, et al: Phase I dose escalation study of MK-0457, a novel Aurora kinase inhibitor, in adult patients with advanced solid tumors. Cancer Chemother Pharmacol 67: 305-314, 2011

19. Gontarewicz A, Balabanov S, Keller G, et al: Simultaneous targeting of Aurora kinases and Bcr-Abl kinase by the small molecule inhibitor PHA-739358 is effective against imatinib-resistant BCR-ABL mutations including T315I. Blood 111: 4355-4364, 2008

20. Arkenau HT, Plummer R, Molife LR, et al: A phase I dose escalation study of AT9283, a small molecule inhibitor of aurora kinases, in patients with advanced solid malignancies. Ann Oncol 23: 1307-1313, 2012

21. Schwartz GK, Carvajal RD, Midgley R, et al: Phase I study of barasertib (AZD1152), a selective inhibitor of Aurora B kinase, in patients with advanced solid tumors. Invest New Drugs: Jun 2 , 2012 (E-pub ahead of print).

22. Aihara A, Tanaka S, Yasen M, et al: The selective Aurora B kinase inhibitor AZD1152 as a novel treatment for hepatocellular carcinoma. J Hepatol 52: 63-71, 2010.

23. Macarulla T, Cervantes A, Elez E, et al: Phase I study of the selective Aurora A kinase inhibitor MLN8054 in patients with advanced solid tumors: safety, pharmacokinetics, and pharmacodynamics. Mol Cancer Ther 9: 2844-2852, 2010.

24. Sehdev V, Peng D, Soutto M, et al: The aurora kinase A inhibitor MLN8237 enhances cisplatin-induced cell death in esophageal adenocarcinoma cells. Mol Cancer Ther 11: 763-774, 2012.
25. Venkataraman S, Alimova I, Tello T, et al: Targeting Aurora Kinase A enhances radiation sensitivity of atypical teratoid rhabdoid tumor cells. J Neurooncol 107: 517-526, 2012

26. Li P, Zhou Q, Ren L and Xiao L: Clinical implication of the expression of Aurora B in normal endometrium and endometrial carcinoma. J Huazhong Univ Sci Technolog Med Sci 337-339, 2008.

27. Kurai M, Shiozawa T, Shih HC, et al: Expression of Aurora kinases A and B in normal, hyperplastic, and malignant human endometrium: Aurora B as a predictor for poor prognosis in endometrial carcinoma. Hum Pathol 36: 1281-1288, 2005.

28. Moreno-Bueno G, Sánchez-Estévez C, Cassia R, et al: Differential gene expression profile in endometrioid and nonendometrioid endometrial carcinoma: STK15 is frequently overexpressed and amplified in nonendometrioid carcinomas. Cancer Res 63: 5697-5702, 2003

29. Yang F, Guo X, Yang G, Rosen DG and Liu J: AURKA and BRCA2 expression highly correlate with prognosis of endometrioid ovarian carcinoma. Mod Pathol 24: 836-845, 2011.

30. Lin YG, Immaneni A, Merritt WM, et al: Targeting aurora kinase with MK-0457 inhibits ovarian cancer growth. Clin Cancer Res 14: 5437-5446, 2008.

31. Beussel S, Hasenburg A, Bogatyreva L, Hauschke D, Werner M and Lassmann S: Aurora-B protein expression is linked to initial response to taxane-based first-line chemotherapy in stage III ovarian carcinoma. J Clin Pathol 65: 29-35, 2012.

32. Zhang L and Zhang S: ZM447439, the Aurora kinase B inhibitor, suppresses the growth of cervical cancer SiHa cells and enhances the chemosensitivity to cisplatin. J Obstet Gynaecol Res 37: 591-600, 2011.

33. Sun C, Chan F, Briassouli P and Linardopoulos S: Aurora kinase inhibition downregulates NF-kappaB and sensitises tumour cells to chemotherapeutic agents. Biochem Biophys Res Commun 352: 220-225, 2007

34. Tao Y, Zhang P, Girdler F, et al: Enhancement of radiation response in p53-deficient cancer cells by the Aurora-B kinase inhibitor AZD1152. Oncogene 27: 3244-3255, 2008. 